\title{
Restoring Vision by Regenerative Medicine Using Stem Cell: A Review
}

Iqbal RK*, Ibrahim M, Shafiq M, Hameed A and Naz G

Institute of Molecular Biology and Biotechnology, Bahauddin Zakariya University, Multan-60800, Pakistan

\begin{abstract}
Restoring vision is the most important center of attention of our research program, On the other hand, to attain our objective and convey the preeminent eminence of stem cell therapy; we have to understand the fundamental biology of stem cells. (RPE) Retinal Pigmented epithelium layer degenerates and contributing to a succession of actions resulting in visual impairments. Regenerative medicine holds promise for the spanning almost for all body system and the eye is an ideal organ to use in research because it is the only part of nervous system that is the visible and easily accessible. This review outlines advances in therapy and the main spotlight for the improvement and advance of cell therapies that are being confronted today.
\end{abstract}

Keywords: Regenerative medicine; Therapeutic transplantation; Photoreceptors; Macular degeneration; Retina deterioration

\section{Introduction}

Regenerative medicine is the recent and emerging branch of science in which replacement of cells, tissues and organs occur with the establishment of function which performs normally. Stem cells require the capability to grow or carry out the process of differentiation into numerous kinds of cells [1]. Main or key component of regenerative medicine is stem cells. It is a very powerful biological tool and potential therapy. Stem cells are of four types On the basis of trans differentiation potential, that is, unipotent, multipotent, pluripotent, and totipotent. On the other hand, the majority of induced pluripotent stem cell also present by name of bioengineered cells in the umbilical cord of embryo and adult [2]. Based on regenerative applications, undeveloped cells can be sorted as embryonic foundational microorganisms (ESCs), tissues particular ancestor undifferentiated organisms (TSPSCs), mesenchymal immature microorganisms (MSCs), umbilical rope immature microorganisms (UCSC), and bone marrow undeveloped cells (BMSCs) and iPSCs. Advancement in tissues engineering technologies of this stem cell used in regenerative medicine [3]. The stem cell is the raw material by which human tissue and perhaps someday, whole organs might be regenerated in the lab from single cells. Stem cell regenerative medicine offers the prospects of studying and treating injuries of the eye as never before. In stem cells rest the hopes of millions of medical patients and aspiration of thousands of researchers the world over. The stem cells have the regenerative potential to overcome many distressing eye diseases and other achievable goals include the slow and eventually prevent the loss of sight and to bring back vision for patients who have previously lost sight [4]. Mesenchymal stem cells present in various body fluids and peripheral blood. Cell therapies emerging therapeutic approach with therapeutic action and cell to be delivered to their target region [1]. Optimizing regenerative medicine for eye particularly grow healthier photoreceptor from the human stem cell. Thus identified molecules that help the new cells survive and creating the best environment to keep transplanted cells alive in the eye. For the protection of eye, cornea plays an important role for the fortification of inner sensitive structures of the eye and its visual function is to allow light into the eye. To maintaining the light path tissue in the anterior segment and their interaction are also important [4]. An induced pluripotent stem cell can be used in regenerative medicine at the same time in stem cell therapy. These cells can exist differentiated into various types of cells and miRNAs promote their differentiation. The efficiency and superiority of pluripotent stem cell play a vital role within regenerative medicine [5]. Stem cells have a multifaceted structure with undeveloped and functionally undifferentiated. There are various properties of these cells together with propagation in which they include the capacity to segregate for an unlimited period of time and self-renewal in which cell resembles with parents after division. Photoreceptors and retinal neural cell are the varied types of stem cells [6]. Applications of proceed stem cells based regenerative medicine contain cornea which serves as ideal tissue due to convenience and ease of its structure and support for restoring vision by mean of stem cell therapy [7]. To maintain vision, protection of cells of retina their structures along with covered neural tissues are imperative and for the accomplishment of long interlude vision re-establishment, functional replacements of retinal cells occur [8]. Cornea stromal stem cell brings sight renovation therapy which maintains corneal epithelial cells [9]. The disintegration of photoreceptors for visual perfection and responsible cells which consists of cone photoreceptors leads to visual impairment [10]. Pluripotent stem cells have distinctive ability to discriminate into somatic cells which are the type of an adult human body and the new age of stem cells research for regenerative therapy by mean of pluripotent stem cell improvement successfully [11]. The substitution of a defective part of the body with regenerative medicine applies to organisms to a certain extent than a precise system and re-establishment occur through regenerative medicine. Scratched and unhealthy cells, organ and tissue restore, repair, return and redevelop using regenerative medicine [12]. Regenerative medicine fashioned very hopeful result for regeneration in the series of organs and for vision cornea is translucent and allow the entrance of light into the eye [13]. In case of totally degenerated retina photoreceptors substitute could restore some vision, on the other hand, cell exchange also restore vision in supplementary retina [14]. Cell rehabilitation via cell transplantation

*Corresponding author: Iqbal RK, Institute of Molecular Biology and Biotechnology, Bahauddin Zakariya University, Multan-60800, Pakistan, Tel: +92 61 9210071; E-mail: Khalid.iqbal@bzu.edu.pk

Received October 17, 2018; Accepted December 05, 2018; Published December 11,2018

Citation: Iqbal RK, Ibrahim M, Shafiq M, Hameed A, Naz G (2018) Restoring Vision by Regenerative Medicine Using Stem Cell: A Review. J Biomol Res Ther 7: 161. doi:10.4172/2167-7956.1000161

Copyright: $\odot 2018$ Iqbal RK, et al. This is an open-access article distributed under the terms of the Creative Commons Attribution License, which permits unrestricted use, distribution, and reproduction in any medium, provided the original author and source are credited. 
in which cells consequential from embryonic or pluripotent stem cells in which assorted development stages can be controlled [15]. Generally vision loss occurs due to retinal degenerative disorders, stem cell therapy move toward its cure and eventual goal to bring back vision is the transplantation of stem cell-based retina [16]. Stem cells have likely to make a distinction in response to cytokines that makes these cells eye catching therapeutically and the infinite extent of retinal cells are formed by stem cells. By replacing departed photoreceptors stem cells have the impending to restore vision [17]. On the other hand, another main reason for vision loss is the corneal sightlessness and the greatest cure for restoring vision is the corneal transplantation. Local cornea and cornea tissue both are imperative for vision restoration [18]. In regenerative medicine therapy, pluripotent stem cell used as a functional organ. Progenitor cells also used as a cell type similar to stem cells in regenerative medicine [19]. Stem cell engineering occurs by replacing the detrimental retinal tissue and human developing stem cells develop keen on photoreceptors by transplanting stem cell to stimulate retinal tissues [20]. The invention of regenerative medicine involves cell therapies leading which cell can grow fast with the protected and useful cell-based products [21]. Appropriate orientation is essential for stem cell transplantation because cells delivered in suspension with the several degenerative changes in the treated eye [22]. Photoreceptors are one of the sheets of retinal and loss of retinal photoreceptors take place from a degenerative abnormality. Loss of retinal cells involves harm to the optic nerve and instantaneously loss visual function [23]. Therapeutic transplantation takes place by engineered stem cells which are patient specific with the differentiated cells to substitute scratched tissues to restore vision [24]. Mesenchymal stem cell for progress glaucoma or retinas pigmentosa beneficial used for depression resistant cure of eye [25]. In the case of vision loss, retinal photoreceptors including rods and cones are loss and this degenerative retina replaced by the reintroduction of healthy photoreceptors by cell substitution therapy [26]. Major the reason for vision loss is the retinal disintegration and photoreceptor loss and introduction of photoreceptors by replacement therapy with the demarcation of new photoreceptors improve visual function in retinal tissue [27]. Maintained vision function by preservation therapies includes preserved photoreceptors and the goal of protection is achieved by preservation strategies [28]. Loss of retinal cells takes place by retinal degeneration and autologous stem cell treatment grip great promise intended for a retinal cure. Markers are also present in the retina for the reprogrammed retort to differentiation [29]. Retinal ganglion cells transplanted in the retina which is a modified form of stem cells and induced pluripotent stem to encourage their survival in the eye [30]. Mostly degeneration of photoreceptors occur due to the absence of retinal pigment epithelium leading to vision loss, integration of photoreceptors may improve visual function [31]. Mainstream of stem cell therapy concentrate on embryonic stem cells due to their strong pluripotency. Viral vector organization used for the transplantation of stem cells for stem cells based therapy [32]. Regenerative medicine generally uses mesenchymal stem cells for clinical purpose because diverse prosperities make them attractive for therapeutics and they have the ability to restore vision [33]. Protection of eye structure from environmental injuries and further important functions like light refraction as well as transmission performed by cornea thus it is vital for normal vision [34]. Extreme visual impairment or blindness occurs due to the degeneration of RPE leads to degeneration of photoreceptors. To perform daily life activities vision is essential due to degenerative eye disorders irreversible vision loss [35]. Among cellbased approaches for the restoration of vision photoreceptors replacement is one of them in regenerative medicine, it is the stem cell- based therapy [36]. Retinal pigment epithelium is transplanted into the patient along with fetal retinas for the improvement of vision [37]. Pluripotent stem cells in the human retina are the main center of attention for our stem cell research for the restoration of vision [38].

\section{Retina}

The retina is the photosensitive component of the central nervous system which consists of rods and cones, amercing, bipolar cells, ganglion and support cells [39]. The retina is one most imported structure in the central nervous system. Retinal cells types are distinguished in different layers [40]. Loss of retinal neurons is a common disease. Neuronal cell death is the main cause of vision loss [41]. Disturbance and loss of RPE degrade photoreceptors with macular degeneration. Human developing stem cell performs unlimited sources of RPE cell for transplantation blinding condition [42]. RPE transplantation was first published approximately 20 years since RPE transplantations are used in the treatment of retinal disease [43]. Due to retinal damage, a micro vascular disease that is diabetic retinopathy is caused. DR depends on the entire retinal cell. The experimental manifestation of $\mathrm{RD}$ primarily owes to change in the retina [44]. Vision loss is mostly due to retinal dysfunction. Various stem cells approaches are used to treat retinal disease and control vision loss or blindness [45]. Retinal degeneration is the main cause of the vision loss. These conditions are characterized by the death of the photoreceptors cell in the retina. Photoreceptor transplantation restores the vision [46]. Intravitreal anti-vascular endothelial growth therapy considered for treatment of the retinal disorder [47].

\section{Cornea}

Ocular surface is composed of cornea and conjunctivas which are not only protect the eye but also enable vision. The coronal epithelium is the most important external surface of the cornea damage to this highly specialized structure leads to the vision loss [48]. Diabetes is the main cause of the blindness. It affects the ocular structure and causes complication. Approximately $70 \%$ of diabetes patients have corneal abnormalities and leads to blindness or vision loss [49]. The cornea is an external part of the eye and highly exposed to the ultraviolet radiation. Cornea protects the eye from the UV rays. The UV rays can penetrate the full thickness of the cornea and reach to the lens and damage the eye which causes vision loss or blindness [50]. Corneal epithelium covers eye its high accessibility for stem cells make an excellent model. Corneal epithelium sc is present at the limbus a narrow ring-shaped boundary between cornea and conjunctiva. Limb stem cells serve as a boundary. Loss of limb SC cause deficiency and cannot be repaired and leads to the blindness [51]. The ocular surface is damaged by trauma or disease which leads to blindness. In this respect, the traditional treatment or cornea transplantation repair the vision loss. Neurotrophic keratopathy is a corneal disease which is caused due to absent corneal sensation and innervations. In the absence of innervations causes blindness Clinical trials suggest that renovation of cornea improve cornea sensation and maintain vision with NK [52]. Keratoconus and postoperative keratectasia is the common cause of blindness. To treat these disease corneal collagen cross-linking used as the tool and cause regression [53]. Corneal sensations protect the eye from injury. Corneal nerves consist of neuromediators which are necessary for corneal epithelium maintenance and healing. Patient who lack NK cause ulceration and cause blindness [54] the restoration of clear vision is present in a patient who has corneal blindness and has poor corneal transplantations techniques. These patients fail to have keratoplasties due to injury or autoimmune disease and have limb stem 
cell deficiencies. The Boston keratoprosthesis is a corneal device that is used to restore vision [55].

\section{Age Related Macular Degeneration}

Macular disintegration is an ailment that affects macular part of the retina and the main reason for vision loss in AMD [56]. 2014 the widespread presence of AMD was about $8.7 \%$. An estimated that 169 million people in 2020 and 288 million in 2040 with AMD [57] the most vigorous form is nonvascular age-related macular degeneration, which is responsible for rapid vision loss [58]. Drugs with the intention of treat AMD have been discovering, therefore, rejection for dry form. Several challenges so as to affected retinal tissue with ADM are not easy to obtain. Refusal animal model that impersonate the disease and human trails costly and long [59]. In 2001 eye disease learn to explore assembly record random results that a dose antioxidant vitamins plus zing formation effecting in control of vision loss [60]. The presences of druses which are related focal hypo pigmentation and hyper pigmentation of RPE increase the chance of progressive advanced to $\mathrm{AMD}$ and have the potential for vision loss [61]. AMD cause blindness, treatment for AMD is absent due to unawareness of macular events Lipid which circulates in the macula has an important factor in the development of the disease. These lipids participate in tissue injury which can induce innate immune responses [62]. CRISPER Cas9 is responsible for the treatment of genetic disease. Vegfa gene specific cas 9 ribonucleoprotein is injected into the retinal pigment epithelium [63]. Choroidal nonvascular grounds vision loss by macular degeneration and Intravitreal vascular endothelial development aspect treats the NVAMD [64].

\section{Vision Repair by Photoreceptor Relocation}

The retinal degenerative diseases consist of Retinitis pigmentosa (RP), age-related macular degeneration (AMD), this disease is hereditarily varied and composite syndrome by 50 dissimilar genomic hazard loci recognized to epoch [65]. 19 from 50 encounter whole genome significance [66]. Initial AMD classically grants in the sixth span of a lifetime or advanced with the development of lipid identical pledges named drusen that gather underneath the RPE and drusen alike pledges that seem in space of sub-retina [67] and Stargardt disease (SD). They have different causes and different demographic they are connected with the damage to the photoreceptors (PRs). For the treatment of RP, AMD and SD genetic factor and medication treatment are mostly used but once PRs are damaged they are unable to restore. Recently stem cell therapy is used to treat them.

\section{Photoreceptor Transplantation}

A source is needed that provide the photoreceptors aimed at the cure of the degenerative infection of the retina where photoreceptors are degenerated comprehensively with or without the participation of the RPE. In one transplantation using the hiPSC from the retina, the photoreceptors of the human that direct the GFP beneath the switch of interphotoreceptor retinoid required protein receptor persisted separated via FACS then inserted cutting-edge empty portion of sub retina of wild-type mice [68]. After subretinal injection, few of the photoreceptor from the donor traveled to external nucleus coating as FAC organized hESC derivative photoreceptors. Similarly, photoreceptors obtained from the piggish induced pluripotent stem cells remained considered through virus-related idea (retinal compulsory protein 3 ) then following the chemical induced host retinal stage detected in the ONL at a frequency of $1 \%$ [69]. In contrast to the subretinal inoculation intravitreal addition of iPSC looked fewer operative by stimulating external retina addition [70]. Choroid ECs must also currently been fruitfully produced from iPSCs $[71,72]$. The postmitotic rod precursor secluded from the initial post-delivery rats distinguished hooked on developed poles then developed a synapsis connection with the wild-type rats. Similar contributor pole populace too re-established bright replies cutting-edge the malformed rats [73]. The wanted donor cell population was known and secluded from the reporter line transgenic animals but their use is not suitable for clinical purposes. In these days human photoreceptor-specific cell surface marker which is appropriate for FACS is yet not branded but to enhance aimed at photo-receptor forerunner since phase definite rats developing retina a mixture of the CD73 and CD24 are used. Trendy the development of the actual tactic aimed at external retina restoration construction of populace of the giver photoreceptor after social cause remains single of the numerous necessary stages. Thoughtful besides influencing the mass retina atmosphere is unfavorably a difficult job because it differs markedly based on the kind and phase of the disease. The factors that affect the donor cell existence and incorporation include the reliability of external preventive film, being plus the degree of the glial damaging, plus position of internal retina motherboard and the additional elements [74]. Preclinical readings by means of the RCS rat presented that subretinal relocation of humanoid NPCs resulting from humanoid prenatal cortex caused in the extended-period liberation of the visual task $[75,76]$. It was supposed that their activities associated with their capability phagocytosis photoreceptor external sections as well as additional trophic properties [77]. Luckily not every retinal illness seems to have complex boundaries to the combination. Deprived of before cure, dawn-organize rats retina relocated through rats slab indications framed a structurally unique benefactor chamber layer that developed hooked on a supposed outside nuclear coating, with the simultaneous rebuilding of bright initiated papillary reactions plus bright-interceded conduct [78]. The honesty of the crowd retina might similarly remain important cutting-edge crowd invulnerable reaction toward benefactor chambers. Subretinal empty place takes remained looked towards remain invulnerable advantaged position cutting-edge sound judgment. Be that as it may, at the point when visual honesty is traded off by RPE misfortune as well as debasement for plasma-retina hindrance, big particle eater outbreak plus microglial plus T-lymphocyte formation start the invulnerable reaction, bringing about unite dismissal [79]. Indeed at the point, once postpartum photoreceptor contributor chambers part insignificant haplotype character through the crowd, an incessant invulnerable reaction can weaken long haul unite survival without invulnerable concealment [80]. Occupied collected, this discoveries underline the important portion for illness particular setting in benefactor chamber existence plus joining, plus suggest this matchless before mostly coordinated allogeneic hiPSC founded cure plan might never remain satisfactory aimed at every single deteriorating sickness. Interestingly, autologous utilization hiPSC chamber composes conveys great probability of going around safe complexities. There are two methods of photoreceptor relocation named as the dissociated photoreceptor cells resulting as a postponement and retina area or micro-cumulative relocation [81].

\section{Transplantation of Dissociated Cells}

Shuffled cells weakened for long duration deprived of advantage vital finding come to know in 2006 after new and directly columnmitotic photoreceptor precursors in preference to retinal progenitor cells (RPC), which are till now going to multiply or developed mature cells remained recognized as the utmost fruitful cell form aimed at relocation. The cells stood accomplished to remain alive for a long period of time, maturing plus pictorial development [82]. Grown-up 
photoreceptors can quiet assimilate nonetheless unimportantly in a small amount and through abridged existence capability in vitro, may be partially linked to the automated and breakdown with the help of enzymes is obligatory [83]. Till now it remained believed that subscriber photoreceptors remained capable to substantially travel and participate into receiver external nuclear coating founded on pieces of training by immune glowing classification of protein that might initiate from the giver. Though, fresh revisions must be recommended that this is a comparatively infrequent occasion in creatures by certain grade of photoreceptor conservation, and in its place here seems to remain certain procedure of cytoplasmic interchange among the giver and receiver cells aimed at the IF discoveries [83]. Initial stage medical judgments with condensed RPE cells manufacturing cilia neurotrophic factors recommended certain photoreceptor defense in diseased person by retina deterioration [84]. As stated before, RPE cells might also use an important influence on photoreceptor restoration and existence by trophic special effects only, assumed mainly to be owing to the manufacture of dye epithelium-derivative factor (PEDF).

Distribution of cells to the subfoveal position needs foveal dispassion, which might negotiate the vision of a person, mainly in the location of retinal degenerative disease. Transfer of NPCs subretinal has, nevertheless, established that they might travel from subretinal injection place fairly comprehensively [85]. Also, on relocation in a rodent ideal of photoreceptor deterioration, these MC-resulting photoreceptor-similar cells were capable to travel and assimilate in host external nuclear layer, prominent to a Development in photoreceptor task as measured by electroretinography [86]. Through overexpression of proneural transcription element Ascll joint with a usage of histone deacetylase inhibitor, which changed the epigenetic Shape of the MC genome, mature mice were capable to produce retinal neurons after MCs in situ afterward injury of the retina [87]. Relocation of these generated allogeneic RPE saved vision into RCS swine into the separation phase dependent way. Especially, relocation of an Intermediary 4-week phase of RPE separation maximum reliably conserved vision matched to mature or newer RPE cells [88]. Afterward, relocation seems to be reliant on host situations mainly in adulthood, with relocating to young retinas existing more fruitful than matured ones [89]. It is likely, nevertheless, that distinction, immigration, and existence might be better by modifications in transfer and host atmosphere [90].

\section{Photoreceptors Recognition}

Having no pigmentation, photoreceptors cells fit to relocation remain fewer relaxed to recognize. The markers between cells applied in the test tube to recognize the photoreceptors like CRX and NRL can't stay applied to kind cells for relocation by glowing or magnet stimulated cell categorization [91]. Readings by animal models must organized photoreceptors by transgenic glowing protein appearance focused by agents of photoreceptor genes though these remain improbable to stay satisfactory for humanoid readings. The cell outward markers CD73 has been acknowledged as markers aimed at the red photoreceptors secluded after the fetal rats [92]. And have remained exposed to upturn cell incorporation whichever lonely or in form of association by CD24 [93].

\section{Combined RPE and Photoreceptors Recognition}

In progressive AMD, both RPE plus photoreceptors required to stay relocated. RPE frequently develop head-to-head to neuron retina in organ models, however not as unbroken coating essential their emerging photoreceptors as happens inside the cells. It might be probable to grow culture situation that inspires RPE development and it had freshly remained exposed that $0.5 \%$ alginate upsurge RPE development lengthwise neuron retina [94]. Cells next to RPE destiny display growing MITF appearance and neuroretinal cells $\mathrm{CHX} 10$ appearance following by cone-rod homeobox protein (Crx) and Improving expression as they grow in photoreceptors. In humanoid PSCs, this procedure receipts numerous months reliant on culture situations by cone markers seeming beforehand rods [95].

Instead, it might remain probable to consume flesh manufacturing to harvest a syndicate relocate of RPE single layer on a biomimetic film by an extra decomposable support for a photoreceptor coating organs derived from the photoreceptor derived sheet. Likewise, configurations together with cells may be written in three-dimensional forms [96]. Certain groups have examined the result of culture situations on RPE distinction, with consuming dissimilar development substrates [97]. The amount of oxygen throughout distinction had also been examined as a probable modulator in cell variation. There is certain proof that hypoxic circumstances throughout stem cell culture carry to promising consequence in neuroectodermal and retinal distinction [98]. IPSC lines differ significantly in their capability to distinguish in RPE and photoreceptors. These alterations might be credited to numerous causes. The difference in endogenous gene appearance governing variation has been exposed to be one of the elements [98].

\section{Retinal Pigment Epithelium Role in Vision Control}

Retinal pigment epithelium (RPE) is a monolayer eye which is imperative for the strength of the photoreceptor cell. Human embryonic stem cell (hESC) used for transplantation of RPE, Age-related macular disintegration (AMD) disease of blindness due to default in RPE and the ideal competitor tissue for curing AMD is only retinal pigment epithelium transplantation [99]. Subretinal surgical treatment shows perforce risk during treat fovea which is intention tissue within AMD. A newly developed system for delivery of cell used in which black of the eye cover with mono injection without the detachment of retina but photoreceptor degeneration take a long time about 5 months to retrieve while human bone substance mesenchymal stem cell (IBMMSCs) intravitreal, these stem cell produced large clamp in vitreous void retinal function retrieve for little extent about 3 months after transplantation [100]. These are four transcriptional factors Oct4, Sox2, Klf4, and c-Myc are enough for reprogramming fibroblast of the mouse into pluripotent stem cell [101]. Stargardt syndrome most widespread reason of AMD in little children, adults, and human embryonic stem cells use regarding that disease [102]. Use of stem cell in retinal disease required less cost easily access and this use becomes the need of today. Stem cells (ESC, iPSC) use in AMD and staggered disease and vision restoring quality increase 25,20 points respectively [6]. The major cause of blindness is age-related macular dystrophy in adult having two type's neovascular AMD (NV-AMD) and non-neovascular AMD (NNV-AMD). If Retinal pigment, Bruch's membrane damaged then it causes complete vision loss in NNV-AMD. Anti VEGF drugs use against NV-AMD [103]. Adult human RPE stem cells use instead of RPE cells in transplantation. RPE transplantation has proven a powerful renewal [104]. Age-related macular degeneration, retinitis pigmentosa are the degenerative disease of retina causes the death of photoreceptor and 60 million people influenced by blindness all over the world Anti-VEGF nowadays is the best therapy for wet AMD [105]. Rat is the model organism for the transplantation of stem cells from different sources [106]. An embryonic and pluripotent stem cell can be developed PRs and RPE cell. PR transplant is able to harmonize with the host retina still in the delayed stage of retinal collapse [107].

\section{Difficulties to RPE Transplantation}

This survey will demonstrate the difficulties looking by the vitro 
retinal specialist and there are four fundamental territories in which specialists confronting challenges:

a) Preferred technique: exterior (transchoroidal) versus interior (transversal)

b) Origin of giver RPE cell

c) Kinds of careful instrumentation

d) Avoidance and control of PVR \& repetitive retinal separation

\section{External Versus Internal: A Careful Approach}

The cornea is the sensitive part of eye expelled, and the globe was open for the kinds of the strategy. Thus, the scientist facing difficulties in reattaching the neurosensory retina [108]. Replacement of strategy for a congested eye technique, consisting of pars plana vitrectomy, retinotomy, and cells conveyance through a pipette, the retinal reattachment was obtained [109]. All of the above investigations, contributor retinal pigment epithelial cells effectively joined with BrMandexternal fragments of photoreceptor phagocytosis could be depicted. various specialists have kept on utilizing an inside transvitreal approach, including standards plana vitrectomy, though others support by using external approach including dismemberment of the transsclerochoroidal subretinal infusion and back sclera of RPE cells. The Peyman was the first who uses the external approach [109]. The outer approach is best in creature considers, particularly in rodents, in which the globe is little, the focal point vast, and there's no obvious vitreous pit to work within [110]. However, this procedure requires a burst of BrM as well as causes choroidal injury, prompting the danger of extreme intraocular or suprachoroidal drain, and may conceivably prompt inflammation and insusceptible reactions that would not happen with the transversal approach. In bigger eyes, the inside careful approach is impressively less demanding [111]. However, this procedure requires a burst of $\mathrm{BrM}$ as well as causes choroidal injury, prompting the danger of extreme suprachoroidal drain, and may conceivably prompt inflammation with insusceptible reactions that would not occur with the transversal approach. In larger eyes, the inside careful comes up tube impressively less demanding.

\section{Sources of cells}

In spite of the fact that the idea of relocation of the cell is to regenerate RPE labor and avert photoreceptor bad luck, the cells which transplanted shouldn't actually be RPE cells. The Sub-retinal mixture of iris shadow epithelial (IPE) cells, Schwann cells, humanoid focal sensory system stem cells [85]. And umbilical line cells altogether inspire photoreceptor bar in the RCS rodent. Many types of the bases of the cell can be employed to deliver an unadulterated cultivated cell in vitro. From the cautious perspective, bases of the cell are able to be separated into subsequent kinds:

a) Autologous retinal coloring epithelium cell

b) Autologous Iris Tincture Epithelium cell

c) In vitro sophisticated allogenic cell

\section{Autologous iris coloring epithelium}

For autologous cell transplantation IPE cells are a hotspot since these are like RPE cells, however, are considerably less demanding to accumulate [112]. Embryonic cell lines are the sources of the retinal pigment epithelial cell and IPE cells, and these cells composes basic polarization and fills in like a boundary to direct the entry of particles and little atoms and to imprison lipids proteins layer dispersion. Essentially, the tight intersection of RPE and IPE is apparently comparative. Approximately 20 weeks the rabbits make up IPE cells in the subretinal space [113]. In IPE cells quality articulation varies from RPE cells. Quality articulation in intracellular and extracellular restricting proteins, that are fundamental for the visible colors digestion, bringing down in vitro IPE cells in comparison to the RPE cells, IPE cell can phagocytosis photoreceptor external portions yet are less ready to corrupt them contrasted and RPE cells [114].

\section{Pluripotent stem cells}

The utilization of foundational microorganism innovation contains guarantee as a unique wellspring of cells for transplantation. Foundational microorganisms can self-renewing property while keeping up a stable in the undifferentiated state, subsequently, separate it indifferent cell write within the body. Human embryonic foundational microorganisms (hESCs) could be secluded from inward blastocyst cell mass at approximately five days pre-treatment. These cells are now able to be kept up vague below in vitro conditions, like the pluripotent cells, and, when needed can be separated into retinal pigment epithelial (RPE) cells [99]. The difficulties regarding human embryonic stem cells can be overwhelmed through prompted pluripotent undifferentiated cells (iPSCs) utilization. In 2012 Nobel Prize granted to Yamanaka for his work [115]. The viral vector system utilizes to embed different key qualities in DNA which at that point responsible for reconstructing of the cell into an undifferentiated organism healthy for delivering any phone genealogy of the three germ layers. As opposed to just being utilized as an alternative source, the utilization of induced pluripotent stem cells in a blend with quality treatment advance helpful potential. For that, as it may, avoidance of the oncogenic factors shown the important or negative impact on the re-creating and incomprehensible in the case of reconstructing itself may prompt tumorigenesis [116].

\section{Removing remaining RPE}

Lopez et al utilized a precious stone cleaned needle yet consequent histological examination uncovered various incidental breaks in $\mathrm{BrM}$, bringing about cell expansion from the choroid into the subretinal space. Utilized for the disengagement and culture of rabbit RPE cells is like that already detailed for human RPE [117]. Eyes reaped from a grown-up pale skinned person and pigmented rabbits were permitted to remain at $4^{\circ} \mathrm{C}$ for around $18 \mathrm{~h}$ to encourage the division of the retina from the RPE. The front portion, vitreous, and retina were expelled and put in eyeglass after that eye hatch at $37 \mathrm{C}$ for 1-2 hour after that by utilization of pipette evacuates the RPE cells. Stenzel et al have as of late detailed utilization of a half $\mathrm{mm}$ prolene circle to encourage RPE evacuation. In rabbits demonstrate a region of two and a half $\mathrm{mm} \times$ one and a half $\mathrm{mm}$ was treated with a solitary upcoming and in reverse stroke. The 70\% territory was observed without RPE cells, instead of the information that a couple of minute BrM slashes and choriocapillaris blood clumps likewise happened. Additional slender, more flexible prolene circle was minor compelling, just like a wire of about $0.1 \mathrm{~mm}$, which likewise caused sub-retinal hemorrhage [104].

\section{Proliferative Vitreo Retinopathy}

Eye injury is an important problem throughout the world. Eye injury as a major health problem affects those people who are lived in industrialized countries. Ocular trauma leads to vision loss or permanent blindness up to millions of people. Retinal detachment is caused by the ocular trauma and often leads to proliferative Vitreoretinopathy [16]. Proliferative Vitreoretinopathy is mostly caused by the treatment failure of retinal detachment [118].

\section{Counteractive action and control of PVR}

PVR is inflammatory and fibrotic progression so as to happen in patients when open retina or during tears. This is due to the relocation 
Citation: Iqbal RK, Ibrahim M, Shafiq M, Hameed A, Naz G (2018) Restoring Vision by Regenerative Medicine Using Stem Cell: A Review. J Biomol Res Ther 7: 161. doi:10.4172/2167-7956.1000161

Page 6 of 8

and expansion of retinal pigment epithelial cells and these cells obtained in framed membranes of PVR. Gathering a fringe RPE-choroid unite appears to create a marginally top rank of PVR and potential purposes behind this incorporate arrival of RPE cells amid fringe collecting, horrible augmentation of the macular retinotomy for join inclusion, or proceeded with the arrival of RPE surgical operations [119]. No self-evident distinction in rate PVR amongst unrivaled and mediocre giver locales, recommending that there is a preferred standpoint from endeavoring to keep away from the inflammatory watery medium in the substandard retina which could not coordinate with tamponade. Albeit a few creators review the utilization of pharmacological operators to anticipate PVR improvement, no one is utilized as a part of casual clinical practice. The silicone oil is used in the process of intraocular tamponade has been all around portrayed in counteractive action and restraint of PVR [120].

\section{Conclusion}

This review article is about the transplantation of eyes through adopting different approaches by using iPCS or embryonic stem cells. A lot of work done on transplantation in past, present and future Regenerative medicine is the most emerging branch of science in which stem cells use to generate the organ in the lab. Adult and embryonic stem cell use used this purpose, iPSC also uses for regeneration of cell. Stem cell therapy uses to treat RP, AMB, SD retinal diseases. Many challenges are present still today in transplantation. Sub-retinal injections used for photoreceptors transplantation in mice. Scientist facing difficulties in reattaching neurosensory retina but some modification into closed eye technique, comprising of pars plana vitrectomy and some other was achieved. External approach use in rodents because have the small lens but the internal approaches are easy and use in human for transplantation in conclusion we can say that by using the stem cells it is possible to recover the vision and may use for treatment of various eyes disease or disorders by applying for regenerative medicine.

\section{References}

1. Potdar PD, Jethmalani YD (2015) Human dental pulp stem cells: applications in future regenerative medicine. World J Stem Cells 7: 839.

2. Roy S, Kern TS, Song B, Stuebe C (2017) Mechanistic insights into pathological changes in the diabetic retina: implications for targeting diabetic retinopathy. American J Path 187: 9-19.

3. Kuriyan AE (2017) Vision loss after Intravitreal Injection of Autologous "Stem Cells" for AMD. New England J Med 37: 1047-1053.

4. Williams R (2018) Biomaterials for Regenerative Medicine Approaches for the Anterior Segment of the Eye. Advanced Health Care Mat 7: 1701328.

5. Zeng ZL, Lin XL, Taan LL, Liu YM (2018) MicroRNAs: Important regulators of induced pluripotent stem cell generation and differentiation. Stem Cell Rev 14: 71-81.

6. Öner A (2018) Stem Cell Treatment in Retinal Diseases: Recent Developments. Turk J Ophthalmol 48: 33-38.

7. Stern JH (2018) Regenerating Eye Tissues to Preserve and Restore Vision. Cell Stem Cell 22: 834-849.

8. Singh R (2018) Pluripotent Stem Cells for Retinal Tissue Engineering: Current Status and Future Prospects. Stem Cell Rev 14: 463-483.

9. Shojaati G (2018) Compressed Collagen Enhances Stem Cell Therapy for Corneal Scarring. Stem cells Trans Med 7: 487-494.

10. Santos-Ferreira (2015) Daylight vision repair by cell transplantation. Stem Cells 33: 79-90.

11. Hu L, Zhao B, Wang S (2018) Stem-Cell Therapy Advances in China. Human Gene Ther 29: 188-196.

12. Stark JF (2018) Perspectives on human regeneration. Palgrave Communi 4: 66.
13. Islam MM (2018) Biomaterials-enabled cornea regeneration in patients at high risk for rejection of donor tissue transplantation. NPJ Regen Med 3: 2

14. Chichagova V, Hallam D, Collin J (2018) Cellular regeneration strategies for macular degeneration: past, present and future. Eye 32: 946-971.

15. Roska B, Sahel JA (2018) Restoring vision. Nature 557: 359.

16. Rathod R (2018) Induced pluripotent stem cells (iPSC)-derived retinal cells in disease modeling and regenerative medicine. J Chem Neuroanatomy.

17. Rao RC, Dedania VS, Johnson MW (2017) Stem cells for retinal disease: a perspective on the promise and perils. American J Ophthalmol 179: 32-38.

18. Palchesko RN, Carrasquilla SD, Feinberg AW (2018) Natural Biomaterials for Corneal Tissue Engineering, Repair and Regeneration. Adv Healthc Mater 7 : 1701434.

19. Javadi S (2018) New Frontiers in Transplantation and Stem Cell Technology: A Brief Synopsis. Open J Organ Transplant Sur 8: 13.

20. Barriga-Rivera A, Suaning GJ (2018) Visual prostheses, optogenetics, stem cell and gene therapies: splitting the cake. Neural Regen Res 13: 805.

21. Marks P, Gottlieb S (2018) Balancing safety and innovation for cell-based regenerative medicine. New Engl J Med 378: 954-959.

22. da Cruz L (2018) Phase 1 clinical study of an embryonic stem cell-derived retinal pigment epithelium patch in age-related macular degeneration. Nature Biotechnol 36: 328

23. Mead B (2017) Concise review: dental pulp stem cells: a novel cell therapy for retinal and central nervous system repair. Stem Cells 35: 61-67.

24. Madl CM, Heilshorn SC, Blau HM (2018) Bioengineering strategies to accelerate stem cell therapeutics. Nature 557: 335.

25. Langrzyk A (2018) Critical view on mesenchymal stromal cells in regenerative medicine. Antioxidants \& Redox Signaling 29: 169-190.

26. Lakowski J (2015) Transplantation of photoreceptor precursors isolated via a cell surface biomarker panel from embryonic stem cell $\square$ derived self $\square$ forming retina. Stem Cells 33: 2469-2482.

27. Kundu J (2018) Interphotoreceptor matrix based biomaterial: impact on human retinal progenitor cell attachment and differentiation. J Biomed Mat Res Part B App Biomat 106: 891-899.

28. Jones MK (2017) Cell-based therapeutic strategies for replacement and preservation in retinal degenerative diseases. Progress in Retinal and Eye Res 58: 1-27.

29. Johnsen EO (2018) Proliferative Cells Isolated from the Adult Human Peripheral Retina only Transiently Upregulate Key Retinal Markers upon Induced Differentiation. Curr Eye Res 43: 340-349.

30. Wu S (2018) Induced Pluripotent Stem Cells Promote Retinal Ganglion Cell Survival After Transplant. Investig Ophthalmol \& Vis Sci 59: 1571-1576.

31. Betz VM (2018) Bone morphogenetic protein-2 is a stronger inducer of osteogenesis within muscle tissue than heterodimeric bone morphogenetic protein-2/6 and-2/7: Implications for expedited gene-enhanced bone repair. $J$ Gene Med 20: e3042.

32. Yang D, Jiang $T$, Lin $P$, Chen $H$, Wang L, et al. (2017) Apoptosis inducing facto gene depletion inhibits zearalenone-induced cell death in a goat Leydig cell line. Reprod Tox 67: 129-139.

33. Hosseini S (2018) Regenerative Medicine Applications of Mesenchymal Stem Cells. Adv Exp Med Biol 7: 43.

34. Gonzalez G (2018) Limbal stem cells: identity, developmental origin, and therapeutic potential. Wiley Interdiscip Rev: Developmen Bio 7: e303.

35. Apatoff MBL (2018) Autologous stem cell therapy for inherited and acquired retinal disease. Regen Med 13: 89-96.

36. Qiang S (2018) Morphological and Genetical changes of endothelia progenitor cells after in-vitro conversion into photoreceptors. J Photochem and Photobiology 183: 127-132.

37. Mandai M (2017) IPSC-derived retina transplants improve vision in rd1 endstage retinal-degeneration mice. Stem Cell Rep 8: 69-83.

38. Achberger K (2018) Stem cell-based retina models. Adv Drug Deliv Rev.

39. Morescalchi F (2013) Proliferative vitreoretinopathy after eye injuries: an 
Citation: Iqbal RK, Ibrahim M, Shafiq M, Hameed A, Naz G (2018) Restoring Vision by Regenerative Medicine Using Stem Cell: A Review. J Biomol Res Ther 7: 161. doi:10.4172/2167-7956.1000161

overexpression of growth factors and cytokines leading to a retinal keloid. Mediators of inflammation 2013: 1-12.

40. Roldán-PM (2012) Modelling postoperative visual acuity with and without proliferative vitreoretinopathy associated with primary rhegmatogenous retinal detachment. Eye 26: 1329.

41. Papastavrou VT, Chatziralli I, McHugh D (2017) Tamponade for Retinectomy in PVR-Related Retinal Detachments: A Retrospective Study. Ophthalmo and Therapy 6: 161-166.

42. Idelson M (2009) Directed differentiation of human embryonic stem cells into functional retinal pigment epithelium cells. Cell Stem Cell 5: 396-408.

43. da Cruz (2007) RPE transplantation and its role in retinal disease. Prog Retinal \& Eye Res 26: 598-635

44. Kady N (2017) Increase in acid sphingomyelinase level in human retina endothelial cells and CD34+ circulating angiogenic cells isolated from diabetic individuals is associated with dysfunctional retinal vasculature and vascular repair process in diabetes. J Clinical Lipidology 11: 694-703.

45. Park SS (2017) Advances in bone marrow stem cell therapy for retina dysfunction. Prog Ret \& Eye Res 56: 148-165.

46. Kicic A (2003) Differentiation of marrow stromal cells into photoreceptors in the rat eye. J Neuro Sci 23: 7742-7749.

47. Lanzetta $P$, Loewenstein A, Committee VAS (2017) Fundamental principles of an anti-VEGF treatment regimen: optimal application of intravitreal antivascular endothelial growth factor therapy of macular diseases. Graefe's Arch Clin Experi Ophthalmol 255: 1259-1273.

48. Bashir H, Seykora JT, Lee V (2017) Invisible shield: review of the cornea epithelium as a barrier to UV radiation, pathogens and other environmental stimuli. J Ophthalmic \& Vision Research 12: 305

49. He J (2017) Recovery of corneal sensitivity and increase in nerve density and wound healing in diabetic mice after PEDF plus DHA treatment. Diabetes 66 2511-2520.

50. Delic NC (2017) Damaging effects of ultraviolet radiation on the cornea. Photochem and Photobio 93: 920-929.

51. Nasser W, Amitai-Lange A, Soteriou D, Hanna R, Tiosano B, et al. (2018) Corneal-committed cells restore the stem cell pool and tissue boundary following injury. Cell Rep 2: 323-331.

52. Catapano J, Antonyshyn K, Borschel GH (2018) Corneal Neurotization Improves Ocular Surface Health And Prevents Corneal Scarring In A Rat Mode of Neurotrophic Keratopathy. Plast Reconstr Surg Glob Open 6: 18-19.

53. Lee HK, Ryu JS, Jeong HJ, Kim MK, Oh JY, et al. (2018) Protection of Corneal Limbus from Riboflavin Prevents Epithelial Stem Cell Loss after Collagen Cross-Linking. J Ophthalmol 2018: 1-7.

54. Catapano J, Fung SM, Halliday W, Jobst C, Cheyne D (2017) Documentation Of Improved Ocular Surface Health After Corneal Neurotisation And Reinnervation Using Magnetoencephalography And Histology. Plastic \& Reconstru Surg Global Open 5: 10-11.

55. Lin M (2017) Vision retention in early versus delayed glaucoma surgical intervention in patients with Boston Keratoprosthesis type 1. PloS one 12 e0182190.

56. Mulyukov Z, Weber S, Pigeolet E, Clemens A, Lehr T, et al. (2018) Neovascular age-related macular degeneration-a visual acuity model of natural disease progression and ranibizumab treatment effect. CPT: Pharmacomet \& Sys Pharma 7: 660-669.

57. Balaratnasingam C, Messinger JD, Sloan KR (2017) Histologic and optical coherence tomographic correlates in drusenoid pigment epithelium detachment in age-related macular degeneration. Ophthalmol 124: 644-656.

58. Anderson G (2018) An Induced Pluripotent Stem Cell Patient Specific Model of Complement Factor $\mathrm{H}(\mathrm{Y} 402 \mathrm{H})$ Polymorphism Displays Characteristic Features of Age-Related Macular Degeneration and Indicates a Beneficial Role for UV Light Exposure. Stem Cells 36: 626-626.

59. Chew EY, Clemons TE, Agron E (2013) Long-Term Effects of Vitamins C E, Beta-Carotene and Zinc on Age-Related Macular Degeneration. AREDS Report No 35 2017: 1604-1611.

60. Rao P (2018) Real-World Vision in Age-Related Macular Degeneration Patients Treated with Single Anti-VEGF Drug Type for 1 Year in the IRIS Registry. Ophthalmol 125: 522-528.
61. Wilde C (2017) Prevalence of age-related macular degeneration in an elderly UK Caucasian population-The Bridlington Eye Assessment Project: a crosssectional study. Eye 31: 1042.

62. Handa JT, Cano M, Weing L, Datta S, Liu T, et al. (2017) Lipids, oxidized lipids, oxidation-specific epitopes, and age-related macular degeneration. Biochimica et Biophysica Acta 1862: 430-440

63. Kim K, Park SW, Kim JH, Lee Sh, Kim D, et al. (2017) Genome surgery using Cas9 ribonucleoproteins for the treatment of age-related macular degeneration. Genome Res 27: 419-426.

64. De Croos FC (2017) Treat-and-extend therapy using aflibercept for neovascular age-related macular degeneration: a prospective clinical trial. American Ophthalmol 180: 142-150.

65. Gorin MB (2012) Genetic insights into age-related macular degeneration: controversies addressing risk, causality, and therapeutics. Mol Asp Med 33 467-486.

66. Fritsche LG (2013) Seven new loci associated with age-related macular degeneration. Nature Genetics 45: 33-43.

67. Curcio CA, Messinger JD, Sloan KR, McGwain G, Spaide RF, et al. (2013) Subretinal drusenoid deposits in non-neovascular age-related macular degeneration: morphology, prevalence, topography, and biogenesis model. Retina 33: 265-276.

68. Lamba DA (2010) Generation, Purification and Transplantation of Photoreceptors Derived from Human Induced Pluripotent Stem Cells. PloS one 5: e8763.

69. Akimoto M (2006) Targeting of GFP to newborn rods by Nrl promoter and temporal expression profiling of flow-sorted photoreceptors. Proceed Nat Acad Sci 103: 3890-3895.

70. Parameswaran S (2010) Induced pluripotent stem cells generate both retinal ganglion cells and photoreceptors: Therapeutic implications in degenerative changes in glaucoma and age related macular degeneration. Stem Cells 28 695-703.

71. Songstad AE (2015) Generating iPSC-derived choroidal endothelial cells to study age-related macular degeneration. Investig Ophthalmol \& Vis Sci 56: 8258-8267.

72. Songstad AE (2017) Connective Tissue Growth Factor Promotes Efficient Generation of Human Induced Pluripotent Stem Cell $\square$ Derived Choroidal Endothelium. Stem Cells Trans Med 6: 1533-1546.

73. MacLaren RE (2006) Retinal repair by transplantation of photoreceptor precursors. Nature 444: 203-207.

74. Barber AC (2013) Repair of the degenerate retina by photoreceptor transplantation. Proceed Nati Acad Sci 110: 354-359.

75. Gamm DM (2007) Protection of visual functions by human neural progenitors in a rat model of retinal disease. PloS one 2: e338.

76. Wang S, Girman S, Lu B, Bischoff N, Holmes T, et al. (2008) Long-term vision rescue by human neural progenitors in a rat model of photorecepto degeneration. Investig Ophthalmol \& Visual Sci 49: 3201-3206.

77. Cuenca N, McGill TJ, Lu B, Wang S, Lund R, et al. (2013) Phagocytosis of photoreceptor outer segments by transplanted human neural stem cells as a neuroprotective mechanism in retinal degeneration. Investigat Ophthalmol \& Vis Sci 54: 6745-6756

78. Singh MS, Issa PC, Butler R, Martin C, MacLaren RE, et al. (2013) Reversa of end-stage retinal degeneration and restoration of visual function by photoreceptor transplantation. Proceed Nati Acad Sci 110: 1101-1106.

79. Streilein JW (2002) Immunobiology and privilege of neuronal retina and pigment epithelium transplants. Vision Res 42: 487-495.

80. West EL (2010) Long-term survival of photoreceptors transplanted into the adult murine neural retina requires immune modulation. Stem cells 28: 19972007.

81. Seiler MJ, Aramant RB (2012) Cell replacement and visual restoration by retinal sheet transplants. Prog Ret Eye Res 31: 661-687.

82. Pearson R, Gonzalez C, West EL, Smith AJ, Cristante E, et al. (2016) Donor and host photoreceptors engage in material transfer following transplantation of post-mitotic photoreceptor precursors. Nat Communi 7: 13029. 
Citation: Iqbal RK, Ibrahim M, Shafiq M, Hameed A, Naz G (2018) Restoring Vision by Regenerative Medicine Using Stem Cell: A Review. J Biomol Res Ther 7: 161. doi:10.4172/2167-7956.1000161

Page 8 of 8

83. Gust J, Reh TA (2011) Adult donor rod photoreceptors integrate into the mature mouse retina. Investig Ophthalmol \& Visual Sci 52: 5266-5272.

84. Rossmiller B, Mao H, Lewin AS (2012) Gene therapy in animal models of autosomal dominant retinitis pigmentosa. Mol Vision 18: 2479.

85. McGill TJ, Cottam B, Lu Bin, Wang S, Girman S, et al. (2012) Transplantation of human central nervous system stem cells-neuroprotection in retinal degeneration. European J Neuro Sci 35: 468-477.

86. Jayaram H (2014) Transplantation of photoreceptors derived from human Müller glia restore rod function in the $\mathrm{P} 23 \mathrm{H}$ rat. Stem Cells Transl Med 323-333.

87. Jorstad NL (2017) Stimulation of functional neuronal regeneration from Müller glia in adult mice. Nature 548: 103-107.

88. Davis RJ, Alam NM, Zhao C, Muller C, Saini JS, et al. (2017) The developmental stage of adult human stem cell-derived retinal pigment epithelium cells influences transplant efficacy for vision rescue. Stem Cell Rep 9: 42-49.

89. Abud M (2015) The effect of transient local anti-inflammatory treatment on the survival of pig retinal progenitor cell allotransplants. Trans Vision Sci \& Tech 4: 6.

90. Ballios BG, Cooke MJ, Donaldson L, Coles BLK, Shoichet MS, et al. (2015) A hyaluronan-based injectable hydrogel improves the survival and integration of stem cell progeny following transplantation. Stem Cell Rep 4: 1031-1045.

91. Collin J, Mellough CB, Dorgau B, Przyborski S, Lako M, et al. (2016) Using zinc finger nuclease technology to generate $\mathrm{CRX}$-reporter human embryonic stem cells as a tool to identify and study the emergence of photoreceptors precursors during pluripotent stem cell differentiation. Stem cells 34: 311-321.

92. Lakowski J, Han YT, Pearson RA, Gonzalez-Cordero A, West EL, et al. (2011) Effective transplantation of photoreceptor precursor cells selected via cell surface antigen expression. Stem Cells 29: 1391-1404.

93. Dominic E, Sandra S, Kai P, Denis C, Marius A, et al. (2011) Increased integration of transplanted CD73-positive photoreceptor precursors into adult mouse retina. Investig Ophthalmol \& Vis Sci 52: 6462-6471

94. Hunt NC, Hallam D, Karimi A, Chen J, Lako M, et al. (2017) 3D culture of human pluripotent stem cells in RGD-alginate hydrogel improves retinal tissue development. Acta Biomaterialia 49: 329-343.

95. Mellough CB, Collin J, Khazim M, White K, Lako M, et al. (2015) IGF-1 signaling plays an important role in the formation of three-dimensional laminated neural retina and other ocular structures from human embryonic stem cells. Stem Cells 33: $2416-2430$.

96. Lorber B, Hsiao WK, Martin KR (2016) Three-dimensional printing of the retina Curr Opin Ophthalmol 27: 262.

97. Sorkio A, Heidi H, Kai K, Hannu U, Kati JU, et al. (2014) Structure and barrie properties of human embryonic stem cell-derived retinal pigment epithelia cells are affected by extracellular matrix protein coating. Tissue Eng Part $A$ 20: $622-634$

98. Garita-HernÁndez M (2013) Hypoxia increases the yield of photoreceptors differentiating from mouse embryonic stem cells and improves the modeling of retinogenesis in vitro. Stem Cells 31: 966-978.

99. Carr AJF, Matthew JKS, Conor MR, Michael BP, Lyndon da C, et al. Development of human embryonic stem cell therapies for age-related macular degeneration. Trends Neuro Sci 36: 385-395.

100. Tzameret (2015) Epiretinal transplantation of human bone marrow mesenchymal stem cells rescues retinal and vision function in a rat model of retinal degeneration. Stem Cell Res 15: 387-394.
101. Lu X, Zhao T (2013) Clinical therapy using IPSCs: hopes and challenges. Gen Proteo Bioinforma 11: 294-298.

102. Mehat MS (2018) Transplantation of Human Embryonic Stem Cell-Derived Retinal Pigment Epithelial Cells in Mac Degener Ophthalmology.

103. Nazari H (2015) Stem cell based therapies for age-related macular degeneration: the promises and the challenges. Prog Retin Eye Res 48: 1-39.

104.Stanzel BV (2014) Human RPE stem cells grown into polarized RPE monolayers on a polyester matrix are maintained after grafting into rabbit subretinal space. Stem Cell Rep 2: 64-77.

105.Zhang K, Zhang L, Weinreb RN (2012) Ophthalmic drug discovery: nove targets and mechanisms for retinal diseases and glaucoma. Nat Rev Drug Discov 11: 541.

106. Luo J (2014) Human retinal progenitor cell transplantation preserves vision. J Biol Chem.

107.Zarbin M (2016) Cell-based therapy for degenerative retinal disease. Trends Mol Med 22: 115-134

108. Alexander P (2015) Retinal pigment epithelium transplantation: concepts, challenges and future prospects. Eye 29: 992

109. Peyman G (1975) Biopsy of human scleral-chorioretinal tissue. Invest Ophthalmol Vis Sci 14: 707-710.

110. Stein JD (2009) Adverse events after pars plana vitrectomy among medicare beneficiaries. Arch Ophthalmol 127: 1656-1663.

111. Thumann G (2009) The in vitro and in vivo behaviour of retinal pigment epithelial cells cultured on ultrathin collagen membranes. Biomat 30: 287-294.

112. Abe T (2007) Iris pigment epithelial cell transplantation for degenerative retinal diseases. Prog Retin Eye Res 26: 302-321.

113. Crafoord S (2002) Photoreceptor survival in transplantation of autologous iris pigment epithelial cells to the subretinal space. Acta Ophthalmol Scand 80 387-394.

114. Zeeburg EJ (2012) A free retinal pigment epithelium-choroid graft in patients with exudative age-related macular degeneration: results up to 7 years. Am J Ophthalmol 153: 120-127.

115. Takahashi K, Yamanaka S (2006) Induction of pluripotent stem cells from mouse embryonic and adult fibroblast cultures by defined factors. Cell 126 663-676

116. Zhao T (2011) Immunogenicity of induced pluripotent stem cells. Nature 474: 212.

117. Rosenfeld PJ (2006) Ranibizumab for neovascular age-related macular degeneration. N Engl J Med 355: 1419-1431.

118. Mumm JS (2005) Laminar circuit formation in the vertebrate retina. Prog Brain Res 147: 155-169.

119. Zeeburg EJ, Maaijwee K, Meurs JC (2014) There is no relation between the occurrence of proliferative vitreoretinopathy and the location of the donor site after transplantation of a free autologous retinal pigment epithelium-choroid graft. Acta Ophthalmol 92: 228-231.

120. Alexander $P$ (2008) Prevention and control of proliferative vitreoretinopathy: primary retinal detachment surgery using silicone oil as a planned two-stage procedure in high-risk cases. Eye 22: 815. 